This is an electronic reprint of the original article. This reprint may differ from the original in pagination and typographic detail.

Author(s): Suhonen, Jouni

Title: Nuclear matrix elements for resonant neutrinoless double electron capture

Year: $\quad 2012$

Version:

Please cite the original version:

Suhonen, J. (2012). Nuclear matrix elements for resonant neutrinoless double electron capture. In 12th International Conference on Topics in Astroparticle and Underground Physics (TAUP 2011) 5-9 September 2011, Munich, Germany (pp. 42026). Institute of Physics. Journal of Physics: Conference Series, 375.

https://doi.org/10.1088/1742-6596/375/1/042026

All material supplied via JYX is protected by copyright and other intellectual property rights, and duplication or sale of all or part of any of the repository collections is not permitted, except that material may be duplicated by you for your research use or educational purposes in electronic or print form. You must obtain permission for any other use. Electronic or print copies may not be offered, whether for sale or otherwise to anyone who is not an authorised user. 
Nuclear matrix elements for resonant neutrinoless double-electron capture

This content has been downloaded from IOPscience. Please scroll down to see the full text.

2012 J. Phys.: Conf. Ser. 375042026

(http://iopscience.iop.org/1742-6596/375/4/042026)

View the table of contents for this issue, or go to the journal homepage for more

Download details:

IP Address: 130.234.75.141

This content was downloaded on 15/01/2016 at 10:00

Please note that terms and conditions apply. 
12th International Conference on Topics in Astroparticle and Underground Physics (TAUP 2011) IOP Publishing Journal of Physics: Conference Series 375 (2012) 042026

\title{
Nuclear matrix elements for resonant neutrinoless double-electron capture
}

\author{
Jouni Suhonen \\ Department of Physics, P.O. Box 35 (YFL), FI-40014 University of Jyväskylä, Finland \\ E-mail: jouni.suhonen@phys.jyu.fi
}

\begin{abstract}
The resonant neutrinoless double electron-capture (R0 $\nu \mathrm{ECEC}$ ) is discussed from the point of view of the resonance condition and the involved nuclear matrix elements. A brief outline of the involved theory framework is given. The present status of measurements of the involved atomic masses and possible R $0 \nu$ ECEC candidates is reviewed.
\end{abstract}

\section{Introduction}

At present the neutrinoless double beta $(0 \nu \beta \beta)$ decay is considered to be the most easily accessible means of extracting information on the possible Majorana mass of the neutrino. The reason for this is that the $0 \nu \beta \beta$ decay occurs only if the neutrino is a massive Majorana particle. The search for the $0 \nu \beta \beta$ decay is mostly concentrated on the $0 \nu \beta^{-} \beta^{-}$decays due to their favorable $Q$ values [1]. Assuming that the neutrino carries Majorana mass also the positron emitting modes of $0 \nu \beta \beta$ decay, $0 \nu \beta^{+} \beta^{+}, 0 \nu \beta^{+} \mathrm{EC}$ and $0 \nu \mathrm{ECEC}$, are possible but they are hard to detect owing to their small decay $Q$ values [2]. The neutrinoless double electron-capture, $0 \nu$ ECEC, can only be realized as a resonant decay [3] or as a radiative process with or without a resonance condition [4]. The resonant $0 \nu \mathrm{ECEC}$ decay (R0 $\nu \mathrm{ECEC}$ ) has attracted a lot of experimental attention recently since the resonance condition - close degeneracy of the initial and final (excited) atomic states - can enhance the decay rate by a factor as large as $10^{6}[3]$.

Nuclear-structure calculations associated with the R0 $\nu$ ECEC processes were performed for ${ }^{112} \mathrm{Sn}$ in $[3,5]$, for ${ }^{74} \mathrm{Se}$ in [6] and for ${ }^{136} \mathrm{Ce}$ in [7]. In these calculations the non-relativistic Schrödinger wave functions of the involved electron orbitals were used. Later in [8] the R0 $\nu$ ECEC decay of ${ }^{106} \mathrm{Cd}$ was discussed and the corresponding nuclear matrix element was calculated. In [9] a rough estimate for the nuclear matrix element corresponding to the R0 $\nu$ ECEC decay of ${ }^{152} \mathrm{Gd}$ to the ground state of ${ }^{152} \mathrm{Sm}$ was given.

\section{Brief outline of the theory framework}

In this work we study a particular type of neutrinoless double beta decay, namely the resonant $0 \nu$ ECEC process of the form

$$
e^{-}+e^{-}+(A, Z) \rightarrow(A, Z-2)^{*} \rightarrow(A, Z-2)+\gamma+2 X,
$$

where the capture of two atomic electrons leaves the final atom in an excited state that decays by one or more nuclear gamma-rays and the atomic vacancies are filled by outer electrons with emission of X-rays. The decay is shown schematically in Fig. 1, left panel. On the right panel 
12th International Conference on Topics in Astroparticle and Underground Physics (TAUP 2011) IOP Publishing Journal of Physics: Conference Series 375 (2012) 042026

some possible $0 \nu 2 \beta$ transitions from the atomic ground state of ${ }^{106} \mathrm{Cd}$ to various excited nuclear states in ${ }^{106} \mathrm{Pd}$ have been indicated by horizontal arrows. The R0 $\nu$ ECEC transition involves a high-lying $0^{+}$state (spin-parity assignment is uncertain at the moment) at $2766.26 \mathrm{keV}$ of excitation where the two atomic K-hole energies have been added.

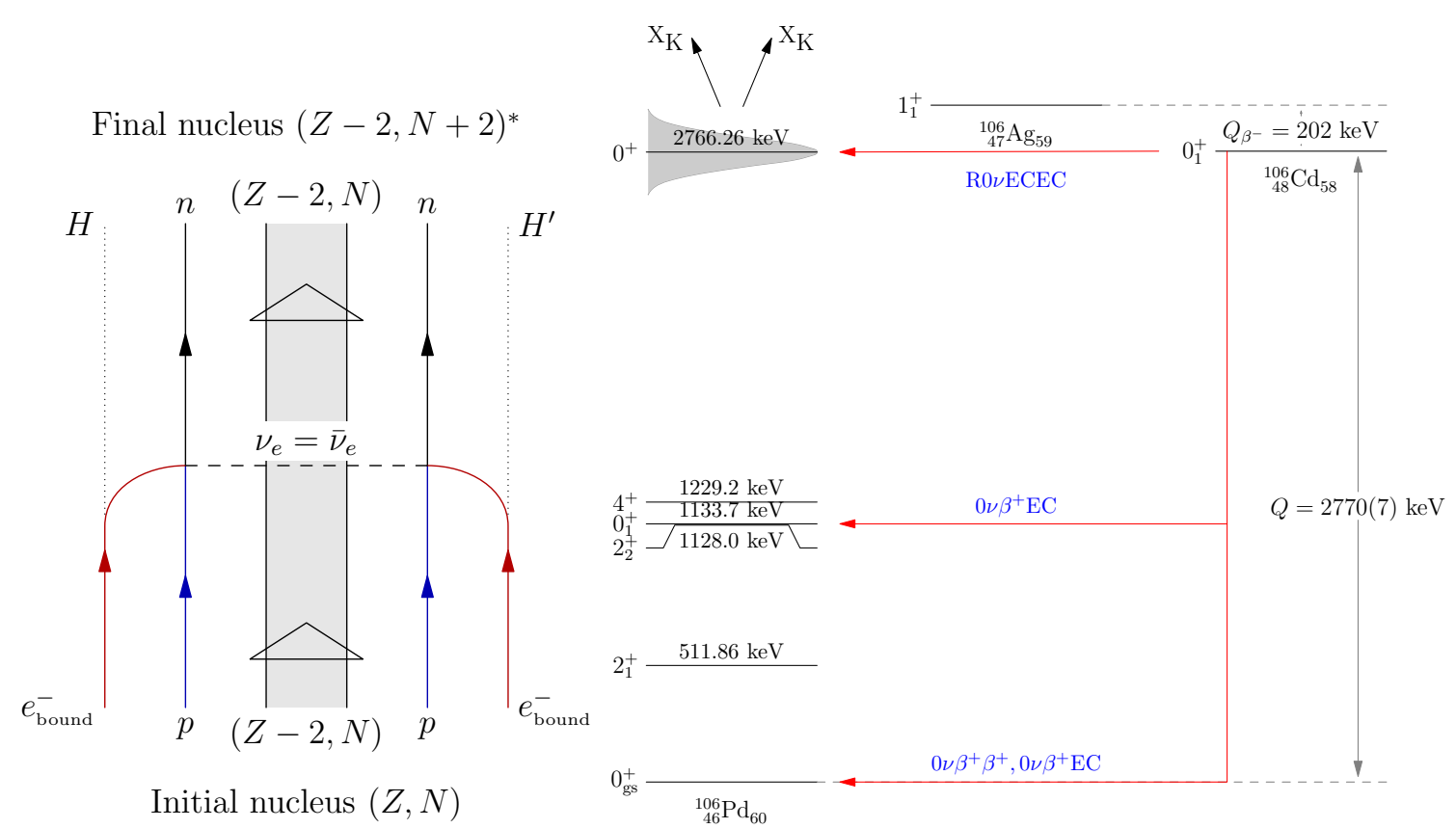

Figure 1. Left panel: decay of the ground state of the initial atom to an excited state of the final atom through the Majorana-neutrino mediated R0 $\nu$ ECEC process. Right panel: Experimental low-energy spectrum of ${ }^{106} \mathrm{Pd}$. The R $0 \nu$ ECEC transition and other possible $0 \nu 2 \beta$ transitions are indicated by horizontal arrows. Also the approximate $Q$ value of the ground-state transition has been given.

The daughter state $(A, Z-2)^{*}$ is a virtual state with energy

$$
E=E^{*}+E_{H}+E_{H^{\prime}}+E_{H H^{\prime}},
$$

including the nuclear excitation energy and the binding energies of the two captured electrons. The last term accounts for the Coulomb repulsion between the two holes. For the half-life of the parent atom the resonance condition can be written [3] in the Breit-Wigner form

$$
\frac{\ln 2}{T_{1 / 2}}=\frac{|\mathcal{M}|^{2}\left\langle m_{\nu}\right\rangle^{2}}{(Q-E)^{2}+\Gamma^{2} / 4} \Gamma
$$

where $\mathcal{M}$ contains the leptonic phase space and the nuclear matrix element, $\Gamma$ denotes the combined nuclear and atomic radiative widths (few tens of electron volts [10]) and $Q-E$ is the so-called degeneracy parameter containing the energy (2) of the virtual final state and the difference between the initial and final atomic masses (the $Q$ value of R0 $\nu \mathrm{ECEC}$ ). The quantity $\left\langle m_{\nu}\right\rangle$ is the effective neutrino mass and it is a linear combination of the light Majorana-mass eigenstates, Majorana-CP phases and electron-flavor elements of the neutrino-mixing matrix [2]. This is the key quantity that one wants to extract from the results of double-beta-decay experiments. 
12th International Conference on Topics in Astroparticle and Underground Physics (TAUP 2011) IOP Publishing Journal of Physics: Conference Series 375 (2012) 042026

To be able to estimate the half-life we have to evaluate the quantity $\mathcal{M}$ in (3). In the mass mode of R0 $\nu$ ECEC it can be written as

$$
\mathcal{M}=G_{0 \nu}^{\mathrm{ECEC}} M_{0 \nu}^{\mathrm{ECEC}},
$$

where $G_{0 \nu}^{\mathrm{ECEC}}$ is the leptonic phase-space factor

$$
G_{0 \nu}^{\mathrm{ECEC}}=\left(\frac{G_{\mathrm{F}} \cos \theta_{\mathrm{C}}}{\sqrt{2}}\right)^{2} \frac{g_{\mathrm{A}}^{2}}{2 \pi} m_{\mathrm{e}}^{3} \mathcal{N}_{0,-1}^{2} \eta_{x}, \quad R_{A}=1.2 A^{1 / 3},
$$

with $G_{\mathrm{F}}$ the Fermi coupling constant, $\theta_{\mathrm{C}}$ the Cabibbo angle, $g_{\mathrm{A}}=1.25$ the axial-vector weak coupling constant, $m_{\mathrm{e}}$ the electron rest mass and $\mathcal{N}_{0,-1}^{2}$ is the normalization of the relativistic K-shell $\left(1 \mathrm{~s}_{1 / 2}\right)$ Dirac wave function for a uniformly charged spherical nucleus. The quantity $\eta_{x}$ in (5) is a suppression factor depending on the atomic orbitals where the two electrons are captured from. Solution of the Schrödinger equation for a point-charge nucleus produces the values

$$
\eta_{\mathrm{KK}}=1, \quad \eta_{\mathrm{KL}_{1}}=\frac{1}{\sqrt{8}}, \quad \eta_{\mathrm{L}_{1} \mathrm{~L}_{1}}=\frac{1}{8}, \quad \eta_{\mathrm{L}_{2} \mathrm{~L}_{2}}=\eta_{\mathrm{L}_{2} \mathrm{~L}_{3}}=\eta_{\mathrm{L}_{3} \mathrm{~L}_{3}}=\frac{1}{96} .
$$

This approximation is reasonable and compares well with the Dirac solution for a homogeneously charged spherical nucleus [11]. The quantity $M_{0 \nu}^{\mathrm{ECEC}}$ is the nuclear matrix element and details of its calculation will be elucidated elsewhere.

\section{Present status of resonant neutrinoless double electron-capture decays}

Table 1 lists the best known cases of R0 $\nu$ ECEC transitions in various nuclei where $Q$-value measurements have been conducted recently. These $\mathrm{Q}$ values have been measured exploiting the Penning-trap techniques. In the cases of ${ }^{96} \mathrm{Ru}$ and ${ }^{106} \mathrm{Cd}$ the assignment of $0^{+}$spin-parity to the resonant state is uncertain.

Table 1. R0 $\nu$ ECEC decay transitions with degeneracy parameters $Q-E$ derived from $Q$-value measurements of the last column. Also the involved atomic orbitals have been given.

\begin{tabular}{ccccl}
\hline Transition & $J_{f}^{\pi}$ & $Q-E[\mathrm{keV}]$ & Atomic orbitals & Ref. \\
\hline${ }^{74} \mathrm{Se} \rightarrow{ }^{74} \mathrm{Ge}$ & $2^{+}$ & 2.23 & $\mathrm{~L}_{2} \mathrm{~L}_{3}$ & {$[6]$} \\
${ }^{96} \mathrm{Ru} \rightarrow{ }^{96} \mathrm{Mo}$ & $2^{+}$ & $8.92(13)$ & $\mathrm{L}_{1} \mathrm{~L}_{3}$ & {$[12]$} \\
& $0^{+} ?$ & $-3.90(13)$ & $\mathrm{L}_{1} \mathrm{~L}_{1}$ & $Q$ from $[12]$ \\
${ }^{102} \mathrm{Pd} \rightarrow{ }^{102} \mathrm{Ru}$ & $2^{+}$ & $75.26(36)$ & $\mathrm{KL}_{3}$ & {$[13]$} \\
${ }^{106} \mathrm{Cd} \rightarrow{ }^{106} \mathrm{Pd}$ & $0^{+} ?$ & 8.39 & $\mathrm{KK}$ & $Q$ from $[13]$ \\
& $(2,3)^{-}$ & $-0.33(41)$ & $\mathrm{KL}_{3}$ & {$[13]$} \\
${ }^{112} \mathrm{Sn} \rightarrow{ }^{112} \mathrm{Cd}$ & $0^{+}$ & -4.5 & $\mathrm{KK}$ & {$[5]$} \\
${ }^{136} \mathrm{Ce} \rightarrow{ }^{136} \mathrm{Ba}$ & $0^{+}$ & -11.67 & $\mathrm{KK}$ & {$[7]$} \\
${ }^{144} \mathrm{Sm} \rightarrow{ }^{144} \mathrm{Nd}$ & $2^{+}$ & $171.89(87)$ & $\mathrm{KL}_{3}$ & {$[13]$} \\
${ }^{152} \mathrm{Gd} \rightarrow{ }^{152} \mathrm{Sm}$ & $0_{\mathrm{gs}}^{+}$ & $0.91(18)$ & $\mathrm{KL}_{1}$ & {$[9]$} \\
${ }^{156} \mathrm{Dy} \rightarrow{ }^{156} \mathrm{Gd}$ & $1^{-}$ & $0.75(10)$ & $\mathrm{KL}_{1}$ & {$[14]$} \\
& $0^{+}$ & $0.54(24)$ & $\mathrm{L}_{1} \mathrm{~L}_{1}$ & {$[14]$} \\
& $2^{+}$ & $0.04(10)$ & $\mathrm{M}_{1} \mathrm{~N}_{3}$ & {$[14]$} \\
${ }^{162} \mathrm{Er} \rightarrow{ }^{162} \mathrm{Dy}$ & $2^{+}$ & $2.69(30) \mathrm{keV}$ & $\mathrm{KL}_{3}$ & {$[12]$} \\
${ }^{168} \mathrm{Yb} \rightarrow{ }^{168} \mathrm{Er}$ & $\left(2^{-}\right)$ & $1.52(25) \mathrm{keV}$ & $\mathrm{M}_{1} \mathrm{M}_{3}$ & {$[12]$} \\
\hline
\end{tabular}

In the case of ${ }^{106} \mathrm{Cd}$ the computed nuclear matrix element [8] with the degeneracy parameter value $Q-E=8.29 \mathrm{keV}$ yields a range of $\mathrm{R} 0 \nu \mathrm{ECEC}$ half-life values:

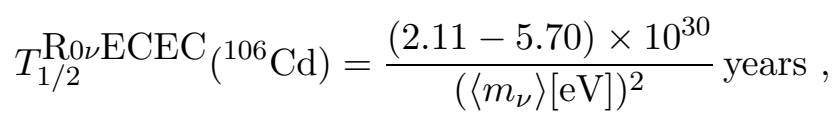


12th International Conference on Topics in Astroparticle and Underground Physics (TAUP 2011) IOP Publishing Journal of Physics: Conference Series 375 (2012) $042026 \quad$ doi:10.1088/1742-6596/375/4/042026

where the effective neutrino mass should be given in units of eV. For ${ }^{112} \mathrm{Sn}$ one obtained in [5]

$$
T_{1 / 2}^{\left.\mathrm{R} 0 \nu \mathrm{ECEC}_{(112} \mathrm{Sn}\right)}>\frac{5.9 \times 10^{29}}{\left(\left\langle m_{\nu}\right\rangle[\mathrm{eV}]\right)^{2}} \text { years }
$$

and for ${ }^{74} \mathrm{Se}$ in $[6]$

$$
T_{1 / 2}^{\mathrm{R} 0 \nu \mathrm{ECEC}}\left({ }^{74} \mathrm{Se}\right) \approx \frac{5 \times 10^{43}}{\left(\left\langle m_{\nu}\right\rangle[\mathrm{eV}]\right)^{2}} \text { years }
$$

In the case of ${ }^{74} \mathrm{Se}$ the astronomically long half-life shown above is explained by the p-wave capture of the two electrons and by the measly nuclear matrix element emerging from the recoil corrections of the weak nucleonic current.

For ${ }^{136} \mathrm{Ce}$ one obtained in [7]

$$
T_{1 / 2}^{\mathrm{R} 0 \nu \mathrm{ECEC}}\left({ }^{136} \mathrm{Ce}\right)>\frac{2.3 \times 10^{33}}{\left(\left\langle m_{\nu}\right\rangle[\mathrm{eV}]\right)^{2}} \text { years }
$$

and for ${ }^{152} \mathrm{Gd}$ in $[9]$

$$
T_{1 / 2}^{\left.\mathrm{R} 0 \nu \operatorname{ECEC}_{(152} \mathrm{Gd}\right)} \approx \frac{10^{26}}{\left(\left\langle m_{\nu}\right\rangle[\mathrm{eV}]\right)^{2}} \text { years } .
$$

In all the above cases the decay rates are suppressed by the rather sizable magnitude of the degeneracy parameter. There are much more favorable cases listed in Table 1 but the associated nuclear matrix elements are still waiting for their evaluation.

\section{Conclusions}

The theoretical and experimental studies of rare weak-interaction processes in nuclei are in a position to shed light on fundamental properties of neutrinos and their mixing. Thus calculation of the associated nuclear matrix elements is an issue of high relevance. In the present work the resonant neutrinoless double electron-capture processes were addressed and a review of the present status of measurements and calculations was given.

\section{Acknowledgments}

This work was supported by the Academy of Finland under the Finnish Center of Excellence Program 2006-2011 (Nuclear and Accelerator Based Program at JYFL).

\section{References}

[1] Avignone III F T, Elliott S R and Engel J 2008 Rev. Mod. Phys. 80 481-516

[2] Suhonen J and Civitarese O 1998 Phys. Rep. 300 123-214

[3] Bernabeu J, De Rujula A and Jarlskog C 1983 Nucl. Phys. B 223 15-28

[4] Sujkowski Z and Wycech S 2004 Phys. Rev. C $\mathbf{7 0} 052501$

[5] Rahaman S, Elomaa V, Eronen T, Hakala J, Jokinen A, Kankainen A, Rissanen J, Suhonen J, Weber C and Äystö J 2009 Phys. Rev. Lett. 103042501

[6] Kolhinen V, Elomaa V, Eronen T, Hakala J, Jokinen A, Kortelainen M, Suhonen J and Äystö J 2010 Phys. Lett. B 684 17-21

[7] Kolhinen V S, Eronen T, Gorelov D, Hakala J, Jokinen A, Kankainen A, Rissanen J, Suhonen J and Äystö J 2011 Phys. Lett. B $\mathbf{6 9 7} 116-120$

[8] Suhonen J 2011 Phys. Lett. B 701 490-495

[9] Eliseev et al S 2011 Phys. Rev. Lett. 106052504

[10] Crasemann B 1975 Atomic Inner-Shell Processes (New York: Academic Press)

[11] Doi M and Kotani T 1992 Prog. Theor. Phys. 87 1207-1231

[12] Eliseev et al S 2011 Phys. Rev. C 83038501

[13] Goncharov et al M 2011 Phys. Rev. C 83038501

[14] Eliseev et al S 2011 Phys. Rev. C $84012501(\mathrm{R})$ 